\title{
Caregiver Perspective on Use of the Specific Carbohydrate Diet In Children with Autism Spectrum Disorder
}

\begin{abstract}
Keywords: Autism; SCD; Diet therapy; Diarrhea; Constipation; Abdominal pain

Abstract

The Specific Carbohydrate Diet (SCD) intervention is a tool that caregivers of children with Autism Spectrum Disorder (ASD) often use to manage gastrointestinal (GI) symptoms as well as related behavioral symptoms. By eliminating certain foods and relying on specific carbohydrates, Gl symptoms frequently improve. Limited data exists on the use and efficacy of this dietary therapy in ASD as well as other diagnoses. The objective of the study was to understand the perspective of caregivers of children with ASD who have used the SCD due to GI problems, behavior, pain, and food selectivity. An anonymous survey was created and conducted on-line. Families were primarily recruited via national ASD and dietary intervention support groups, as well as conferences, other educational events, social media, and several clinics serving children with ASD. A total of 256 participants completed the study: 210 (82\%) were caregivers of male and $46(18 \%)$ were caregivers of female children. The average age of initiation of the diet was 5.77 years and the average time for which the SCD diet was followed was 2.71 years. Data were analyzed using Wilcoxon signed rank and two-sided McNemar's test, where appropriate. The majority of respondents reported significant improvements in Gl symptoms, behavior, pain, food selectivity, and toilet training status after implementing an SCD protocol for a child with ASD. The SCD protocol is used by many families of children with ASD, often without clinica guidance, as an intervention for $\mathrm{Gl}$ and behavioral support. Clinical recommendations must include dietary counseling to ensure that an SCD is warranted and that child's nutritional needs are being met. The majority of caregivers acknowledge benefit of this therapeutic approach and report positive change for each criterion evaluated in this survey. Additional research is warranted on the SCD protocol.
\end{abstract}

\section{Introduction}

Autism Spectrum Disorder (ASD) is comprised of a group development of neurodevelopmental disorders related to differentiated brain development that vary in both complexity and severity. ASD is characterized by impairments in social interaction, deficits in verbal and non-verbal communication and/or the presence of restricted, repetitive and stereotyped patterns of behavior and interests [1]. Originally thought to be a behavioral disorder only affecting development, Gastrointestinal (GI) disorders and associated symptoms including, but not limited to, chronic abdominal pain, constipation, chronic diarrhea and gastro esophageal reflux, are also commonly reported in children with ASD [2-7]. These symptoms are often overlooked due to the child's primary diagnosis of ASD, although it has been recognized that diagnostic and treatment recommendations for the general pediatric population should be considered until the development of evidence-based guidelines specifically for children with ASD $[3,4]$. Although these recommendations are supported by
Nutrition and Health

\section{Barnhill KM*, Richardson W, Rodriguez L, Moreno HT, Devlin M, Sachdev PK and Hewitson L \\ The Johnson Center for Child Health and Development, USA}

\section{*Address for Correspondence}

Barnhill KM, The Johnson Center for Child Health and Development, 1700 Rio Grande, Suite 200, Austin, Texas 78701, USA, Tel: 512.732.8400; Fax: 512.732.8353; E-mail: kbarnhill@johnson-center.org

Submission: 15 February, 2019

Accepted: 18 March, 2019

Published: 29 March, 2019

Copyright: ๑ 2019 Barnhill KM, et al. This is an open access article distributed under the Creative Commons Attribution License, which permits unrestricted use, distribution, and reproduction in any medium, provided the original work is properly cited.

findings in other more recent work the consensus report for evidencebased guidelines is now 8 years old [8], and additional screening tools and treatment options for those with significant GI symptoms and ASD have not really improved.The Specific Carbohydrate Diet (SCD) protocol is a dietary intervention that caregivers of children with ASD frequently employ to address GI symptoms. By eliminating certain foods and relying on specific carbohydrates, multiple GI symptoms often improve. The SCD protocol excludes certain complex carbohydrates from the diet in favor of simpler carbohydrates with a shorter digestion and absorption time to ultimately improve a patient's gastrointestinal status (Supplementary Table S1). The SCD intervention was developed by Dr. Sidney Haas in the 1920s as a treatment for celiac disease [9] and later became a popular approach to treating GI symptoms associated with ASD with the publication of Elaine Gottschall's work, Breaking the Vicious Cycle [10].

In essence, the SCD protocol is grounded in the theory that a dysbiotic environment exists in the GI tract of those with severe symptoms. This can be traced to an overgrowth of harmful bacteria and fungi coupled with a lack of known beneficial bacteria [11]. Three primary consequences of this overpopulation include: (i) the production of an excess of short chain organic acids (thus lowering the $\mathrm{pH}$ of the colon); (ii) an increase in the production of metabolic byproducts of fermentation as well as bacterial toxins; (iii) a possible mutation of some harmless bacteria into pathological forms [10]. The SCD calls for strict avoidance and elimination of all grain-, lactose-, and sucrose-derived carbohydrates from the with the direct aim of restoring and maintaining a healthy intestinal microbial profile [12]. The SCD is an approach that aims to capitalize on awareness of the connection between carbohydrate consumption and growth of an unhealthy intestinal flora by removing this source of energy most utilized by these pathogenic bacteria and fungi. This would in effect reverse or prevent microbial overgrowth. The growth of a more balanced intestinal profile would thus be encouraged [10]. More specifically, it has been determined that certain carbohydrates present more of an issue than others. Complex carbohydrates require additional digestion before they can be absorbed through the intestinal wall. The extra digestive processing required for the proper 
Citation: Barnhill KM, Richardson W, Rodriguez L, Moreno HT, Devlin M, at al. Caregiver Perspective on Use of the Specific Carbohydrate Diet In Children with Autism Spectrum Disorder. J Nutri Health. 2019;5(1): 6.

breakdown of these disaccharides and polysaccharides allows for extra fermentation time in the gut [13]. With this extra fermentation time, bacteria in the intestine derive more energy, reproduce more rapidly, and give off more by-products (toxins) as metabolic waste [11]. It is theorized that these by-products are the trigger for both GI symptoms and behavioral symptoms for children with ASD [14].

While the SCD has gained momentum as an effective intervention for children with Inflammatory Bowel Disease (IBD), ulcerative colitis, and Crohn's disease [12,15-17], its documented use in ASD is limited to a single case study [18]. The objective of this study was to understand the perspective of caregivers of children with an ASD who have implemented an SCD protocol to effect improvement in gastrointestinal problems, behavior, pain, and food selectivity through the utilization of an online questionnaire.

\section{Materials and Methods}

\section{Participants and recruitment}

An online survey was created using surveygizmo.com, and the study was approved by the appropriate Institutional Review Board with expedited review. Caregivers were invited to participate in the study primarily through a number of national and local avenues, including support groups, list-servs, clinics serving those with ASD, and multiple social media strategies. All respondents were aware that they were participating in a research study and gave consent to participate on-line before completing the questionnaire anonymously. Inclusion criteria were as follows: caregiver of a male or female child, 2-18 years of age at the time of initiation of the SCD; the child must have a prior diagnosis of ASD from a licensed professional; and the caregiver must have previously or currently implemented an SCD protocol. Exclusion criteria included children who had a diagnosis of any genetic disorders of metabolism, such as phenylketonuria, leukodystrophy, lysosomal disorder and Wilson's disease or a diagnosis of any genetic syndromes such as Down's syndrome, Angelman Syndrome, Prader Willi, Rett's Syndrome, and Fragile X Syndrome. The survey was open for 7 weeks from July 2016 to September 2016. It was conducted confidentially via surveygizmo. com, who have extensive experience in developing HIPPA-compliant features such that they meet all mandated guidelines.

\section{Study design}

The SCD Questionnaire (SCDQ) is an online survey completed by the primary caregiver of children with ASD. The survey is designed to assess gastrointestinal problems in children with ASD and evaluate how those symptoms may have improved or changed after using this dietary approach. The SCDQ asks questions regarding GI symptoms the child experienced (based on the Rome III Diagnostic Criteria for Functional Gastrointestinal Disorders), pain due to possible GI issues, food selectivity, behavior, and compliance with SCD, as well as collecting demographic data. Caregivers were asked to rank their child's response for each outcome being measured, both before and after implementing the diet. The full SCDQ is provided as Supplementary Table S2.

\section{Data analysis}

Surveys were completed by caregivers of children with a prior diagnosis of ASD who had implemented an SCD protocol. Data was captured by and downloaded from surveygizmo.com. Descriptive analysis of the data was performed using SPSS (Version 22, Armonk, NY: IBM Corporation; 2013). All demographic and clinical variables were summarized using frequencies and percentages for categorical variables. The study employed a repeated-measures design, where the primary aim was to evaluate whether there was change in ASDrelated outcomes after an SCD intervention. The responses to survey questions regarding GI symptoms and associated behaviors were based on a Likert scale with the four options of none, mild, moderate and severe for each outcome measured. These survey responses were ordinal in nature. A numeric rank was given to each response starting from none ranked as 0 , mild as 1 , moderate as 2 and severe as 3 . SPSS was used to conduct a two-tailed Wilcoxon signed-rank $t$ test to calculate the differences between the median of the ranks before and after implementation of the SCD intervention. Since multiple Wilcoxon signed rank tests were conducted to test post intervention differences in outcomes, a Bonferroni correction (0.05/10) was used. This was done to reduce probability of Type I error. As a result, for

Table 1: Demographic data reported by the 256 study respondents.

\begin{tabular}{|c|c|}
\hline Demographic characteristics & $N(\%)$ \\
\hline \multicolumn{2}{|l|}{ Gender } \\
\hline Male & $211(82 \%)$ \\
\hline Female & $45(18 \%)$ \\
\hline \multicolumn{2}{|l|}{ Age (years) } \\
\hline$<5$ & $130(51 \%)$ \\
\hline $5-10$ & $97(38 \%)$ \\
\hline $11-15$ & $25(10 \%)$ \\
\hline $16-18$ & $4(2 \%)$ \\
\hline \multicolumn{2}{|l|}{ Ethnicity } \\
\hline White/Caucasian & $172(67 \%)$ \\
\hline Hispanic/Latino & $32(13 \%)$ \\
\hline Black/African American & $10(4 \%)$ \\
\hline Asian/Pacific Islander & $17(7 \%)$ \\
\hline Native American & $2(1 \%)$ \\
\hline White/Hispanic & $1(0.4 \%)$ \\
\hline Middle Eastern & $2(1 \%)$ \\
\hline Other & $2(1 \%)$ \\
\hline No response & $18(7 \%)$ \\
\hline \multicolumn{2}{|l|}{ Caregiver's education level } \\
\hline Some high school, no diploma & $1(0.4 \%)$ \\
\hline GED/High School & $9(4 \%)$ \\
\hline Trade/technical/vocational training & $11(4 \%)$ \\
\hline Some college, no diploma & $20(8 \%)$ \\
\hline Associate's & $19(7 \%)$ \\
\hline Bachelor's & $84(33 \%)$ \\
\hline Master's & $69(27 \%)$ \\
\hline Doctorate & $14(5 \%)$ \\
\hline Professional Degree & $19(7 \%)$ \\
\hline No response & $10(4 \%)$ \\
\hline \multicolumn{2}{|l|}{ Annual family income (\$) } \\
\hline$<25,000$ & $37(15 \%)$ \\
\hline $25,000-50,000$ & $35(14 \%)$ \\
\hline $50,001-75,000$ & $36(14 \%)$ \\
\hline $75,001-100,000$ & $41(16 \%)$ \\
\hline $100,001-150,000$ & $39(15 \%)$ \\
\hline$>150,000$ & $28(11 \%)$ \\
\hline No response & $40(16 \%)$ \\
\hline
\end{tabular}


Citation: Barnhill KM, Richardson W, Rodriguez L, Moreno HT, Devlin M, at al. Caregiver Perspective on Use of the Specific Carbohydrate Diet In Children with Autism Spectrum Disorder. J Nutri Health. 2019;5(1): 6.

ISSN: $2469-4185$

Table 2: Reported gastrointestinal symptoms before and after SCD intervention.

\begin{tabular}{|c|c|c|}
\hline \multirow{2}{*}{ Variable } & Before SCD & After SCD \\
\hline & $\mathbf{N}(\%)$ & $\mathbf{N}(\%)$ \\
\hline \multicolumn{3}{|c|}{ Abdominal pain } \\
\hline Severe & $31(12 \%)$ & $3(1 \%)$ \\
\hline Moderate & $73(29 \%)$ & $17(7 \%)$ \\
\hline Mild & $72(28 \%)$ & $82(32 \%)$ \\
\hline None & $80(31 \%)$ & $154(60 \%)$ \\
\hline \multicolumn{3}{|c|}{ Constipation } \\
\hline Severe & $45(18 \%)$ & $7(3 \%)$ \\
\hline Moderate & $61(24 \%)$ & $29(11 \%)$ \\
\hline Mild & $59(23 \%)$ & $87(34 \%)$ \\
\hline None & $91(35 \%)$ & $133(52 \%)$ \\
\hline \multicolumn{3}{|l|}{ Diarrhea } \\
\hline Severe & $30(12 \%)$ & $2(1 \%)$ \\
\hline Moderate & $65(25 \%)$ & $19(7 \%)$ \\
\hline Mild & $60(23 \%)$ & $62(24 \%)$ \\
\hline None & $101(39 \%)$ & $173(67 \%)$ \\
\hline \multicolumn{3}{|c|}{ Gastroesophageal reflux } \\
\hline Severe & $15(6 \%)$ & $6(2 \%)$ \\
\hline Moderate & $33(13 \%)$ & $8(3 \%)$ \\
\hline Mild & $36(14 \%)$ & $40(16 \%)$ \\
\hline None & $172(67 \%)$ & $202(79 \%)$ \\
\hline \multicolumn{3}{|l|}{ Gagging } \\
\hline Severe & $10(4 \%)$ & $1(0.4 \%)$ \\
\hline Moderate & $26(10 \%)$ & $9(4 \%)$ \\
\hline Mild & $38(15 \%)$ & $39(15 \%)$ \\
\hline None & $182(71 \%)$ & $207(81 \%)$ \\
\hline \multicolumn{3}{|l|}{ Vomiting } \\
\hline Severe & $8(3 \%)$ & $3(1 \%)$ \\
\hline Moderate & $21(8 \%)$ & $5(2 \%)$ \\
\hline Mild & $35(14 \%)$ & $27(10 \%)$ \\
\hline None & $192(74 \%)$ & $221(85 \%)$ \\
\hline
\end{tabular}

each statistical test a value of $\mathrm{p}<0.005$ was considered statistically significant. Effect sizes were also calculated using the formula $=\mathrm{Z}$ / $(\operatorname{sqrt}(\mathrm{N}))$ where $\mathrm{N}$ was the sum of the observations pre-and postSCD intervention.

A two-sided McNemar's test was conducted to test improvement in two dichotomous outcomes. The first outcome measured improvement in tolerability of foods after SCD intervention. This was assessed by caregiver responses to the question of whether the child could eat more than 15 foods before and after SCD intervention, with the response being either yes or no. The second outcome was to determine whether the child was toilet trained for bowel movements before and after SCD intervention. Again, the response could be either yes or no. To prevent confounding from repeated entries, partially completed surveys were not included in the final analysis for this study.

\section{Results}

A total of 256 complete survey responses were generated and included in this study. There were 65 partially completed responses excluded from the study. The responses represented data collected from the caregivers of 211 (82\%) male and 45 (18\%) female children (Table 1). All respondents reported confirmed diagnoses of ASD, with responses as follows: ASD - 124 (48.4\%); Autism - $72(28 \%)$; Asperger's Syndrome - 20 (8\%); Pervasive Developmental Disorder- not otherwise specified - 32 (13\%); and Autistic Disorder - 8 (3.1\%). Caregivers completing the survey were highly educated. 108/216 (50\%) reported a household annual income above $\$ 75,000$ (Table 1).

The average (SD) age of initiation of the diet was 5.8 (3.3) years, range: 2 to 18.6 years and the average (SD) time for which the SCD diet was followed was 2.7 (2.7) years, range: 30 days to 10.9 years. Survey data reported that 129/256 (50\%) of caregivers began an SCD intervention for their child after recommendation of a practicing physician; 103/256 (40\%) caregivers stated that they sought guidance from a dietitian or nutritionist trained in SCD application prior to beginning this intervention for their child; 141/256 (55\%) caregivers reported that they employed a 48 -hour introductory diet as they initiated the SCD intervention with their child; and 157/256 (61\%) caregivers followed a staged approach to food introduction and menu planning while their child was beginning an SCD. The majority of caregivers reported that they spent six to nine hours of meal preparation per week after implementing the SCD protocol.

Caregivers reported that $163 / 256$ (64\%) children were on a GFCF diet prior to SCD implementation and 26/163 (16\%) of these children had improved constipation, diarrhea, and abdominal pain with the implementation of an SCD protocol. There were 71/256 (28\%) children reported to have participated in feeding therapy prior to SCD. Of these children, caregivers reported that 16/71 (23\%) increased their variety of food intake with implementation of the SCD protocol. Furthermore, 127/256 (50\%) children met criteria for restrictive/ selective eating (fewer than 15 foods) prior to SCD intervention. Of these children, 70/127 (55\%) expanded dietary intake to more than 15 foods after SCD intervention.

The number of children who had severe, moderate, mild or no GI symptoms prior to and after SCD intervention is reported for each GI symptom in Table 2. Abdominal pain, constipation and diarrhea were the most commonly reported concerns. Before SCD intervention, caregivers reported that 104 (41\%) children had moderate to severe abdominal pain. Of those children, 92/104 (89\%) had improved symptoms after SCD implementation. Similarly, 106 (41\%) children had moderate to severe constipation prior to implementing an SCD and of those children, 86/106 (81\%) had improved symptoms after SCD intervention. Finally, 95 (37\%) children had moderate to severe diarrhea prior to implementing an SCD and of those children, 82/95 (86\%) had improved symptoms after SCD intervention.

The number of children who had severe, moderate, mild or no behaviors associated with GI symptoms is reported for each variable in Table 3. Posturing and/or self-injurious behavior was the most commonly reported concern. Before SCD intervention, 131 (51\%) children displayed moderate to severe posturing and self-injurious behavior, and of those children, 114/131 (87\%) had improved symptoms after SCD intervention. Moderate to severe irritability was reported for 141 (55\%) children prior to implementing an SCD protocol, and of those children, 116/141 (82\%) were reported to show improvement after SCD intervention. Moderate to severe anxiety was reported in 150 (59\%) children prior to implementing an SCD, and of those children, 105/150 (70\%) reported improvement after SCD intervention. Finally, before SCD implementation, 120 (47\%) children were not toilet trained for bowel movements. After SCD intervention, 65/120 (54\%) became toilet trained. 
Citation: Barnhill KM, Richardson W, Rodriguez L, Moreno HT, Devlin M, at al. Caregiver Perspective on Use of the Specific Carbohydrate Diet In Children with Autism Spectrum Disorder. J Nutri Health. 2019;5(1): 6.

ISSN: $2469-4185$

Table 3: Reported behaviors associated with GI symptoms before and after SCD intervention.

\begin{tabular}{|c|c|c|}
\hline \multirow{2}{*}{ Behavior } & Before SCD & After SCD \\
\hline & $\mathbf{N}(\%)$ & $\mathbf{N}(\%)$ \\
\hline \multicolumn{3}{|c|}{ Behaviors associated with GI pain (posturing, self-injurious behavior) } \\
\hline Severe & $37(14 \%)$ & $3(1 \%)$ \\
\hline Moderate & $94(37 \%)$ & $30(12 \%)$ \\
\hline Mild & $68(27 \%)$ & $97(38 \%)$ \\
\hline None & $57(22 \%)$ & $126(49 \%)$ \\
\hline \multicolumn{3}{|l|}{ Irritability } \\
\hline Severe & $55(22 \%)$ & $7(3 \%)$ \\
\hline Moderate & $86(34 \%)$ & $53(21 \%)$ \\
\hline Mild & $67(26 \%)$ & $104(41 \%)$ \\
\hline None & $48(19 \%)$ & $92(36 \%)$ \\
\hline \multicolumn{3}{|l|}{ Anxiety } \\
\hline Severe & $54(21 \%)$ & $56(22 \%)$ \\
\hline Moderate & $96(38 \%)$ & $61(24 \%)$ \\
\hline None & $32(13 \%)$ & $13(5 \%)$ \\
\hline \multicolumn{3}{|c|}{ Toilet trained for bowel movements } \\
\hline Yes & $136(53 \%)$ & $201(79 \%)$ \\
\hline No & $120(47 \%)^{\mathrm{a}}$ & $55(22 \%)$ \\
\hline
\end{tabular}

${ }^{\mathrm{a}} 65 / 120(54 \%)$ children who were not toilet trained for bowel movements before SCD intervention became toilet trained after SCD intervention

The level of language before and after SCD intervention is shown in and was reported as follows: non-verbal - 99/256 (39\%), emerging language - 96/256 (38\%), and speaking in sentences - 61/256 (24\%). In previously non-verbal children caregivers reported that language improved a lot $(21 / 161,21 \%)$, moderately $(22 / 161,22 \%)$, a little (23/161, 23\%), or not at all $(33 / 161,33 \%)$ after an SCD intervention (Table 4). In children with emerging language, caregivers reported that language improved a lot $(19 / 96,20 \%)$, moderately $(23 / 96,24 \%)$, a little (19/96, 20\%), or not at all (35/96, 37\%), after SCD intervention. Finally, in children that were reported as speaking in sentences, caregivers reported that language improved a lot (15/61, 25\%), moderately $(11 / 61,18 \%)$, a little $(8 / 61,13 \%)$, or not at all $(27 / 61,24 \%)$ after SCD intervention. Overall, 161 (63\%) caregivers reported that their child's language improved after an SCD intervention (Table 4).

Improvements in GI symptomatology and associated behaviors in children with ASD were analyzed by Wilcoxon signed-rank test (Table 5). A statistically significant reduction in all 10, of the outcomes measured following an SCD intervention were reported. Negative Z-values (median post-test ranks minus median pre-test ranks) were obtained for each outcome. These results were further supported by the $\mathrm{P}$-values $(\mathrm{P}<.005)$, which were significant for all the outcomes. The effect sizes associated with each test ranged from small $(<.1)$ to moderate $(<.3)$ and are reported in Table 5. Finally, a significant increase in the tolerability of foods $(\mathrm{P} \leq .001)$ and in the number of children toilet-trained $(\mathrm{P}=.003)$ were reported after SCD implementation.

\section{Discussion}

While there is a paucity of research evidence speaking to the efficacy of the SCD approach for children with ASD, the SCD is frequently recommended by clinicians and initiated by parents [19]. Given this environment, our goal in this study was to understand caregiver perspectives via an online survey on the implementation of the SCD in children with ASD.
In the 7 weeks that the study was open, we obtained over 250 completed responses to the survey indicating a strong interest in SCD intervention and/or research in the ASD community. Although 50\% of caregivers implemented an SCD intervention for their child after recommendation of a practicing physician, it was apparent that many caregivers did so without any clinical guidance or support. While there are certainly on-line resources that provide meal plans and guidance on the staged-food approach for implementing the SCD, parents should be counseled to work with a dietician or nutritionist familiar with the SCD to ensure their child's nutritional needs are being met.

The majority of caregivers surveyed reported that their child had a positive response to the SCD. Overall, they reported a significant improvement in GI symptoms and related behaviors after implementation of an SCD. The most common GI symptoms reported in participants prior to initiating an SCD included abdominal pain,

Table 4: Level of language reported in 256 children with ASD before and after SCD intervention.

\begin{tabular}{|c|c|}
\hline Level of language/lmprovement & N (\%) \\
\hline Overall improvement in language & $161(63 \%)$ \\
\hline Nonverbal prior to SCD & $99(39 \%)$ \\
\hline and reported language improved a lot while on SCD & $21(21 \%)$ \\
\hline and reported language improved moderately while on SCD & $22(22 \%)$ \\
\hline and reported language improved a little while on SCD & $23(23 \%)$ \\
\hline and reported no improvement in language while on SCD & $33(33 \%)$ \\
\hline Emerging language prior to SCD & $96(38 \%)$ \\
\hline and reported language improved a lot while on SCD & $19(20 \%)$ \\
\hline and reported language improved moderately while on SCD & $23(24 \%)$ \\
\hline and reported language improved a little while on SCD & $19(20 \%)$ \\
\hline and reported no improvement in language while on SCD & $35(37 \%)$ \\
\hline Speaking in sentences prior to SCD & $61(24 \%)$ \\
\hline and reported language improved a lot while on SCD & $15(25 \%)$ \\
\hline and reported language improved moderately while on SCD & $11(18 \%)$ \\
\hline and reported language improved a little while on SCD & $8(13 \%)$ \\
\hline and reported no improvement in language while on SCD & $27(44 \%)$ \\
\hline
\end{tabular}


Citation: Barnhill KM, Richardson W, Rodriguez L, Moreno HT, Devlin M, at al. Caregiver Perspective on Use of the Specific Carbohydrate Diet In Children with Autism Spectrum Disorder. J Nutri Health. 2019;5(1): 6.

ISSN: $2469-4185$

Table 5: Improvements in GI symptomatology and associated behaviors in children with ASD analyzed by Wilcoxon signed-rank test.

\begin{tabular}{|c|c|c|c|}
\hline \multicolumn{1}{|c|}{ Outcome Measure } & \multicolumn{2}{|c|}{ Median post-test ranks minus Median pre-test ranks Statistics } \\
\hline & Z value & $P$-value \\
\hline Abdominal Pain & -9.821 & $P \leq .001$ \\
\hline Constipation & -7.722 & $P \leq .001$ \\
\hline Diarrhea & -8.614 & $P \leq .001$ & -0.434 \\
\hline Gastrointestinal Reflux & -6.08 & $P \leq .001$ \\
\hline Gagging & -5.74 & $P \leq .001$ \\
\hline Vomiting & -4.681 & $P \leq .001$ \\
\hline Throat Pain & -4.514 & $P \leq .001$ \\
\hline Gastrointestinal Pain & -0.381 \\
\hline Gastrointestinal Irritability & -10.279 & $P \leq .001$ \\
\hline Anxiety & -8.207 & $P \leq .001$ \\
\hline
\end{tabular}

aPosturing and/or self-injurious behavior

constipation, and diarrhea. These symptoms were reported as being moderate to severe in $36-40 \%$ of children, consistent with previous studies [20]. After an SCD intervention, the percentage of children reported to have moderate or severe abdominal pain, constipation, and/or diarrhea was reduced to $7-15 \%$. A significant reduction in GI-associated behaviors, including posturing/self-injurious behavior, irritability, and anxiety, was also reported after SCD intervention. The greatest change was seen in GI-associated pain, with caregivers reporting that $50 \%$ of children had moderate to severe symptoms prior to SCD implementation and only $13 \%$ of children were affected with these symptoms after SCD intervention. Overall, these results suggest that an SCD intervention had a positive impact on GIassociated behaviors, as reported by the caregivers participating in our study.

An SCD intervention is frequently utilized for children with IBD and has been reported to provide adequate nutrition [2], although close monitoring is recommended to ensure overall health [17]. In a retrospective study examining the use of an SCD in treating 26 children with IBD, almost $50 \%$ children had a positive effect on both GI symptoms and clinical inflammatory markers, with some children able to discontinue medications and maintain disease control on the SCD alone [15]. Two additional studies, one chart review and one small prospective study, suggest that an SCD may be successful in treating pediatric Crohn's disease $[16,21,22]$. The application of an SCD protocol for treating children with GI disease is not well studied despite being utilized by many people in both the pediatric and general $[15,23]$ population.

GI symptoms and associated behaviors in children with ASD can include abdominal pain, constipation, diarrhea, posturing and self-injurious behavior, as well as increased irritability, anxiety, and poor sleep $[3,24,25]$. In children with little or no language, GI-related behaviors often go undiagnosed leading to increased anxiety and irritability in the child $[4,20,25,26]$. Many children with ASD take longer to become toilet trained than typically-developing children [27]. The application of an SCD to address GI symptoms and associated behaviors in children with ASD is intriguing and requires further study. In our study, many caregivers reported that their child displayed fewer GI-related behaviors and became toilet trained for bowel movements with implementation of an SCD intervention.
An unexpected finding from the survey results was an improvement in language, which was reported for over $60 \%$ of children. This was reported for children with all levels of language whether they were non-verbal, had emerging language, or could speak in sentences. One could surmise that reducing GI symptoms and associated behaviors in children with ASD may allow for better focus in day-to-day activities, less interrupted sleep, and overall better well-being [28,29].

\section{Limitations}

There are several limitations of this study. First, the Specific Carbohydrate Diet Questionnaire (SCDQ) has not been validated for research purposes. Our approach, therefore, cannot clarify whether the GI concerns assessed are reported by caregivers with equal reliability, as some symptoms, such as diarrhea, may be more apparent to caregivers whereas others, such as nausea and pain, may be less obvious and rely more on the child's ability to understand and communicate their internal state. Furthermore, given the nature of using an anonymous reporting instrument, we were unable to verify diagnosis, as well as the adherence to, and compliance with SCD intervention. Furthermore, the study population was not controlled and included some children with reported IBD, and/or other medical complications, as well as children of different ages that followed an SCD for anywhere between 30 days and over 10 years, with and without clinical support, behavioral intervention and/or use of medications. Selection bias must be noted, as respondents were recruited from several SCD support groups, clinics, and autism organizations. Although parents could have underestimated or overestimated actual GI symptoms of their child, parents are generally found to be reliable informants of children's GI problems [30]. Caregivers who responded to the SCDQ may not represent all parents of children with ASD and GI symptoms in their perception of the use of an SCD intervention for children with ASD. Additionally, study respondents were highly educated, with the majority possessing undergraduate or graduate degrees. Finally, the study was designed to capture caregivers' beliefs about their child's responses after implementing an SCD, not objective data and evidence to support that belief. Nevertheless, these responses mirror those we have seen in clinical practice. 
Citation: Barnhill KM, Richardson W, Rodriguez L, Moreno HT, Devlin M, at al. Caregiver Perspective on Use of the Specific Carbohydrate Diet In Children with Autism Spectrum Disorder. J Nutri Health. 2019;5(1): 6.

ISSN: $2469-4185$

\section{Conclusion}

In conclusion, the caregiver survey indicated a strong interest in SCD intervention in the ASD community. The majority of caregivers reported a positive response to SCD in both GI symptoms and related behaviors. Overall, there was a significant improvement in GI symptoms and related behaviors after implementation of an SCD. Given the complexity of dietary interventions, further research is warranted into the mechanism(s) of action and the effectiveness of the SCD on GI and behavioral systems, as well as the impact of this intervention on individuals and families. Research on the implementation of the SCD protocol in ASD would benefit from a larger scale clinical trial to assess changes in the gut microbiota over the time of intervention to assess possible mechanisms of action. Given the incidence of GI symptoms in those with ASD, a dietary evaluation at baseline to assess the impact of diet and GI health on patients with ASD is recommended. Strategies for support of patients attempting SCD are also necessary. This could include provisions for dietitian or nutritionist support for those who are recommended an SCD intervention, including individualized consultation and counseling, as well as professionally-led support groups at the local practice level.

\section{References}

1. American Psychiatric Association (2013) Diagnostic and Statistical Manual of Mental Disorders ( $5^{\text {th }}$ Edn). Arlington, VA: American Psychiatric Publishing

2. Black C, Kaye JA, Jick H (2002) Relation of childhood gastrointestinal disorders to autism: nested case-control study using data from the UK General Practice Research Database. BMJ 325: 419-421.

3. Buie T, Campbell DB, Fuchs GJ 3 ${ }^{\text {rd }}$, Furuta GT, Levy J, et al. (2010) Evaluation, diagnosis, and treatment of gastrointestinal disorders in individuals with ASDs: a consensus report. Pediatrics125 Suppl 1: S1-S18.

4. Buie T, Fuchs GJ $3^{\text {rd }}$, Furuta GT, Kooros K, Levy J, et al. (2010) Recommendations for evaluation and treatment of common gastrointestinal problems in children with ASDs. Pediatrics 125 Suppl 1: S19-S29.

5. Valicenti-McDermott M, McVicar K, Rapin I, Wershil BK, Cohen H, et al. (2006) Frequency of gastrointestinal symptoms in children with autistic spectrum disorders and association with family history of autoimmune disease. J Dev Behav Pediatr 27(2 Suppl) S128-S136.

6. Mannion A, Leader G (2016) An investigation of comorbid psychological disorders, sleep problems, gastrointestinal symptoms and epilepsy in children and adolescents with autism spectrum disorder: A two year follow-up. Res Autism Spect Disord 22: 20-23.

7. Sparks B, Cooper J, Hayes C, Williams K (2018) Constipation in children with Autism Spectrum Disorder associated with increased emergency department visits and inpatient admissions. J Pediatr 202: 194-198.

8. Fulceri F, Morelli M, Santocchi E, Cena H, Del Bianco T, et al. (2016) Gastrointestinal symptoms and behavioral problems in preschoolers with Autism Spectrum Disorder. Dig Liver Dis 48: 248-254.

9. Haas SV, Haas MP (1955) The treatment of celiac disease with the specific carbohydrate diet; report on 191 additional cases. Am J Gastroenterol 23: 344-360.

10. Gottschall E (1994) Breaking the vicious cycle: Intestinal health through diet. The Kirkton Press pp: 205.

11. Strati F, Cavalieri D, Albanese D, De Felice C, Donati C, et al. (2017) New evidences on the altered gut microbiota in autism spectrum disorders. Microbiome 5: 24.

12. Suskind DL, Wahbeh G, Gregory N, Vendettuoli H, Christie D (2014) Nutritional therapy in pediatric Crohn disease: the specific carbohydrate diet. J Pediatr Gastroenterol Nutr 58: 87-91.
13. Turnbaugh PJ, Ridaura VK, Faith JJ, Rey FE, et al. (2009) The effect of diet on the human gut microbiome: a metagenomic analysis in humanized gnotobiotic mice. Sci Transl Med 1: 6ra14.

14. Heberling CA, Dhurjati PS, Sasser M (2013) Hypothesis for a systems connectivity model of Autism Spectrum Disorder pathogenesis: links to gut bacteria, oxidative stress, and intestinal permeability. Med Hypotheses 80: 264-270.

15. Obih C, Wahbeh G, Lee D, Braly K, Giefer M, et al. (2016) Specific carbohydrate diet for pediatric inflammatory bowel disease in clinical practice within an academic IBD center. Nutrition 32: 418-425.

16. Suskind DL, Cohen SA, Brittnacher MJ, Wahbeh G, Lee D, et al. (2018) Clinical and fecal microbial changes with diet therapy in active Inflammatory Bowel Disease. J Clin Gastroenterol 52: 155-163.

17. Braly K, Williamson N, Shaffer ML, Lee D, Wahbeh G, et al. (2017) Nutritional adequacy of the Specific Carbohydrate Diet in pediatric Inflammatory Bowel Disease. J Pediatr Gastroenterol Nutr 65: 533-538.

18. Barnhill K, Devlin M, Moreno HT, Potts A, Richardson W, et al. (2018) Brief Report: Implementation of a Specific Carbohydrate Diet for a child with Autism Spectrum Disorder and Fragile X Syndrome. J Autism Devel Disord.

19. Gogou M, Kolios G (2018) Are therapeutic diets an emerging additional choice in autism spectrum disorder management? World J Pediatr 14: 215223.

20. Neuhaus E, Bernier RA, Tham SW, Webb SJ (2018) Gastrointestinal and psychiatric symptoms among children and adolescents with Autism Spectrum Disorder. Front Psychiatry 9: 515.

21. Wong AP, Clark AL, Garnett EA, Acree M, Cohen SA, et al. (2009) Use of complementary medicine in pediatric patients with inflammatory bowel disease: results from a multicenter survey. J Ped Gastroenterol Nutr 48: 5560

22. Cohen SA, Gold BD, Oliva S, Lewis J, Stallworth A, et al. (2014) Clinical and mucosal improvement with Specific Carbohydrate Diet in pediatric Crohn disease. J Ped Gastroenterol Nut 59: 516-521.

23. Suskind DL, Wahbeh G, Cohen SA, Damman CJ, Klein J, et al. (2006) Patients perceive clinical benefit with the Specific Carbohydrate Diet for Inflammatory Bowel Disease. Dig Dis Sci 61: 3255-3260.

24. Horvath K, Perman JA (2002) Autism and gastrointestinal symptoms. Curr Gastroenterol Rep 4: 251-258.

25. Nikolov RN, Bearss KE, Lettinga J, Erickson C, Rodowski $M$, et al. (2009) Gastrointestinal symptoms in a sample of children with pervasive developmental disorders. J Autism Dev Disord 39: 405-413.

26. Horvath K, Perman JA (2002) Autistic disorder and gastrointestinal disease. Curr Opin Pediatr 14: 583-587.

27. Leader G, Francis K, Mannion A, Chen J (2018) Toileting problems in children and adolescents with parent-reported diagnoses of Autism Spectrum Disorder. J Dev Phys Disabil 30: 307-327.

28. Wasilewska J, Klukowski M (2015) Gastrointestinal symptoms and autism spectrum disorder: links and risks - a possible new overlap syndrome. Pediatric Health Med Ther 6: 153-166.

29. McCue LM, Flick LH, Twyman KA, Xian H (2017) Gastrointestinal dysfunctions as a risk factor for sleep disorders in children with idiopathic autism spectrum disorder: A retrospective cohort study. Autism 21: 1010-1020.

30. Gorrindo P, Williams KC, Lee EB, Walker LS, McGrew SG, et al. (2012) Gastrointestinal dysfunction in autism: parental report, clinical evaluation, and associated factors. Autism Res 5: 101-108

\section{Acknowledgement}

The authors would like to thank all the support staff and clinicians at The Johnson Center for Child Health and Development for assistance with implementing this study. 